\title{
Efficacy and Safety of Low-Dose-Rate Endorectal Brachytherapy as a Boost to Neoadjuvant Chemoradiation in the Treatment of Locally Advanced Distal Rectal Cancer: A Phase-II Clinical Trial
}

\author{
Shapour Omidvari, Shadi Zohourinia ${ }^{1}$, Mansour Ansari, Leila Ghahramani ${ }^{2}$, Mohammad Zare-Bandamiri \\ Ahmad Mosalaei ${ }^{4}$, Niloofar Ahmadloo, Saeedeh Pourahmad ${ }^{5}$, Hamid Nasrolahi ${ }^{1}$, Sayed Hasan Hamedi, \\ Mohammad Mohammadianpanah ${ }^{2}$ \\ Department of Radiation Oncology, Namazi Hospital, Shiraz University of Medical Sciences, Shiraz; ${ }^{1}$ Department of Radiation Oncology, \\ Shiraz University of Medical Sciences, Shiraz; ${ }^{2}$ Colorectal Research Center, Shiraz University of Medical Sciences, Shiraz; ${ }^{3}$ Department of \\ Epidemiology, Kerman University of Medical Sciences, Kerman; ${ }^{4}$ Shiraz Institute for Cancer Research, Shiraz University of Medical Sciences, \\ Shiraz; ${ }^{5}$ Department of Biostatistics, Shiraz University of Medical Sciences, Shiraz, Iran
}

Purpose: Despite advances in rectal cancer treatment over the last decade, local control and risk of late side effects due to external beam radiation therapy (EBRT) remain as concerns. The present study aimed to investigate the efficacy and the safety of low-dose-rate endorectal brachytherapy (LDRBT) as a boost to neoadjuvant chemoradiation for use in treating locally advanced distal rectal adenocarcinomas.

Methods: This phase-II clinical trial included 34 patients (as the study arm) with newly diagnosed, locally advanced (clinical T3-T4 and/or N1/N2, M0) lower rectal cancer. For comparative analysis, 102 matched patients (as the historical control arm) with rectal cancer were also selected. All the patients were treated with LDRBT (15 Gy in 3 fractions) and concurrent chemoradiation (45-50.4 Gy). Concurrent chemotherapy consisted of oxaliplatin $130 \mathrm{mg} / \mathrm{m}^{2}$ intravenously on day 1 plus oral capecitabine $825 \mathrm{mg} / \mathrm{m}^{2}$ twice daily during LDRBT and EBRT.

Results: The study results revealed a significant differences between the study arm and the control arm in terms in the pathologic tumor size $(2.1 \mathrm{~cm}$ vs. $3.6 \mathrm{~cm}, \mathrm{P}=0.001)$, the pathologic tumor stage $(35 \% \mathrm{~T} 3-4$ vs. $65 \% \mathrm{~T} 3-4, \mathrm{P}=0.003)$, and the pathologic complete response $(29.4 \%$ vs. $11.7 \%, \mathrm{P}<0.028)$. Moreover, a significantly higher dose of $\mathrm{EBRT}(\mathrm{P}=0.041)$ was found in the control arm, and a longer time to surgery was observed in the study arm $(\mathrm{P}<0.001)$. The higher rate of treatment-related toxicities, such as mild proctitis and anemia, in the study arm was tolerable and easily manageable.

Conclusion: A boost of LDRBT can optimize the pathologic complete response, with acceptable toxicities, in patients with distal rectal cancer.

Keywords: Rectal neoplasms; Adenocarcinoma; Brachytherapy; Neoadjuvant treatment; Surgery

Received: April 6, 2015 - Accepted: June 25, 2015

Correspondence to: Mohammad Mohammadianpanah, M.D.

Colorectal Research Center, Department of Radiation Oncology, Shiraz

University of Medical Sciences, Shiraz 71936, Iran

Tel: +98-71-36125170, Fax: +98-71-36474320

E-mail:mohpanah@sums.ac.ir

(c) 2015 The Korean Society of Coloproctology

This is an open-access article distributed under the terms of the Creative Commons Attribution NonCommercial License (http://creativecommons.org/licenses/by-nc/3.0) which permits unrestricted noncommercial use, distribution, and reproduction in any medium, provided the original work is properly cited.

\section{INTRODUCTION}

Colorectal cancer is one of the most prevalent leading causes of cancer deaths worldwide [1]. In Asia, the rectum accounts for a higher proportion $(40 \%-50 \%)$ of colorectal cancer primary sites compared to the West $[2,3]$. The majority of rectal cancers present at stages II-III and need standard surgeries, such as a low anterior resection (LAR) and an abdominoperineal resection (APR) [4]. Surgical resection is the treatment of choice for rectal cancer; 
however, large proportions of patients are locally advanced at the time of diagnosis and require adjuvant local and systemic treatment. Neoadjuvant chemoradiation, followed by a total mesorectal excision with either a LAR or an APR, is the current standard of care for patients with stage II-III resectable rectal cancer $[4,5]$.

Despite advances in rectal cancer treatment over the last decade, local control and risk of late side effects due to external beam radiation therapy (EBRT) remain as concerns [6, 7]. A rectal adenocarcinoma is a radiosensitive neoplasm, and radiation dose escalation can potentially optimize local tumor control. Given the circumferential growth pattern of most rectal cancers, endocavitary brachytherapy can potentially enhance local tumor control [8]. Brachytherapy allows delivery of localized radiation that may limit toxicity and allow dose escalation. This mode of radiation therapy can deliver higher doses of radiation to the tumor and adjacent perirectal lymph nodes without any significant increase in toxicity related to normal surrounding tissues, such as the small bowel and the urinary bladder $[9,10]$. Therefore, brachytherapy can be safely applied as a boost to optimize the dose delivered in neoadjuvant, adjuvant or in definitive settings [8]. There is paucity of information in the literature regarding the role of neoadjuvant endorectal brachytherapy as a boost to EBRT in treating patients with rectal cancer. Moreover, the majority of the reports that have examined the use of brachytherapy to treat patients with rectal cancer are retrospective studies $[8,11,12]$. Some studies have also investigated the definitive role of high-dose-rate (HDR) endocavitary or interstitial brachytherapy in treating lower rectal cancer, particularly early lesions [13-15]. The present study aims to investigate the efficacy and the safety of low-doserate endorectal brachytherapy (LDRBT) as a boost to neoadjuvant chemoradiation for treating patients with locally advanced distal rectal cancer.

\section{METHODS}

\section{Patients and preliminary evaluation}

This phase-II clinical trial included 34 patients (study arm) with distal rectal cancer. The patients' performance statuses were scored according to the Eastern Cooperative Oncology Group (ECOG) performance scaling system. Eligible patients had to have a newly diagnosed, locally advanced (clinical T3-T4 and/or N1/N2, M0) rectal adenocarcinoma, no prior therapy, ECOG performance scale score $\leq 1$, and normal or acceptable kidney, liver, cardiovascular, and bone marrow functions. Only patients with rectal tumors located $10 \mathrm{~cm}$ below the anal verge were included, and those having rectal tumors at an upper rectal or rectosigmoid location were excluded. Additionally, patients with metastatic disease at presentation or previous history of pelvic irradiation were excluded. The study was approved by the Clinical Research Ethics Committee of Shiraz University of Medical Sciences in accordance with the code of ethics of the World Medical Association (Declaration of Helsinki) for experiments involving humans. In addition, written informed consents were obtained from all the patients before the trial.

Tumor staging was performed using the seventh edition of the American Joint Committee on Cancer TNM staging system [16]. Clinical staging was performed using imaging studies for all the patients before starting neoadjuvant intervention. Preliminary evaluations involved recording a comprehensive history and performing physical examinations, including careful rectal examinations, colonoscopy, complete blood cell count analyses, liver and renal function studies, measurements of the carcinoembryonic antigen level, chest, abdominal, and pelvic multidetector computed tomography scans, and pelvic magnetic resonance imaging and/or endorectal ultrasonography. For comparative analysis, 102 patients (historical control arm) with rectal cancer were selected. The control arm patients were matched according to sex, age, tumor size, tumor stage, and tumor location (distance from the anal verge), EBRT technique, and concurrent chemotherapy regimen. The pathologic complete response (PCR) rate and the treatmentrelated toxicity were the primary and the secondary endpoints of the study, respectively.

\section{Brachytherapy}

LDRBT was carried out in an outpatient setting by using the Solectron remote system after the machine had been loaded with a cesium-137 sources. All patients but three received LDRBT before chemoradiation. We considered this sequence for evaluating the effect of LDRBT on tumor pain and bleeding. The remaining three patients received brachytherapy once a week after completion of chemoradiation because the severity of their obstructive tumor prohibited insertion of the rectal applicator. The patients were treated in the lateral position. The patients with painful lesions were sedated by using intravenous midazolam $(0.5 \mathrm{mg})$, or rarely by using general anesthesia, before insertion of the rectal. All the patients received a total dose of $15 \mathrm{~Gy}$ in three fractions within 10 days (two fractions per week). The dose was prescribed at $0.5 \mathrm{~cm}$ from the applicator's surface. In this study, we used rectal cylinder applicators that were $2 \mathrm{~cm}$ in diameter with three different lengths, $7.5,10$, and $12.5 \mathrm{~cm}$. With the use of treatment-planning software, the endorectal applicators were loaded to cover the tumor and 2 $\mathrm{cm}$ above and below the tumor edge. Additionally, all the patients received a cycle of concurrent chemotherapy using oxaliplatin at a dose of $130 \mathrm{mg} / \mathrm{m}^{2}$ intravenously on day 1 plus capecitabine at a dose of $1,000 \mathrm{mg} / \mathrm{m}^{2}$ twice daily for 14 days (CAPEOX regimen) during the course of brachytherapy.

\section{External beam chemoradiation}

Concurrent neoadjuvant chemoradiation consisted of conventional EBRT administered by using photons from a megavoltage linear accelerator. The energies of the used photon energy were 6 MV in three-field technique (one direct posterior field and two lateral fields) and $18 \mathrm{MV}$ in the two-field technique (anteroposterior and posteroanterior parallel opposing fields). All the patients 
in the study arm and the control arm were treated in the prone position with a full bladder to reduce small bowel toxicity. A median dose of 45 Gy (range, 45-50.4 Gy) was delivered via a daily fraction of 1.8-2 Gy, with five fractions per week. Concurrent chemotherapy consisted of oxaliplatin at a dose of $130 \mathrm{mg} / \mathrm{m}^{2}$ intravenously on day 1 plus oral capecitabine at a dose of $825 \mathrm{mg} /$ $\mathrm{m}^{2}$ twice daily during the whole period of pelvic radiotherapy with weekend breaks. Two weeks after the completion of radiation, another cycle of chemotherapy (CAPEOX regimen) was administered and subsequently, all the patients were referred for surgery with a median of a 4 - to 8-week interval after the last session of radiation therapy. Acute treatment-related toxicities, including radiation dermatitis, proctitis, noninfective cystitis, and bone marrow suppression, were recorded according to the $\mathrm{Na}$ tional Cancer Institute (NCI) Common Terminology Criteria for Adverse Events ver. 4.0.

\section{Surgical technique}

A standard curative surgery through a total mesorectal excision was performed as a part of a LAR or an APR procedure for all the patients. A colorectal surgeon performed all the rectal surgeries. The decision to perform sphincter-preserving surgery (LAR) was based on the proximity of the tumor to the anal sphincter, the status of the preoperative sphincter function, the clinical response to neoadjuvant therapy, and the patient's preference. Tumors with an adequate distal margin were treated with sphincter-preserving surgery through LAR and a stapled or hand-sewn coloanal anastomosis. After removal of the rectum, colonic J-pouch and coloanal anastomosis procedures were performed. A temporary diverting loop ileostomy or colostomy was performed in all the patients to protect the anastomosis. However, the patients with poor preoperative sphincter function, inadequate distal margin, or involvement of the external anal sphincter were managed by using an APR and permanent colostomy. The pathologic response was assessed after curative surgery. The PCR was defined as the disappearance of all invasive tumors, either on a macroscopic or a microscopic scale, in the rectum and the lymph nodes.

\section{Statistics}

Statistical analyses were performed using IBM SPSS Statistics ver. 19.0 (IBM Co., Armonk, NY, USA). Chi-square, Fisher exact, and Mann-Whitney tests were used for comparing the clinical and the pathological response rates and the categorical clinicopathologic characteristics of the study arm and of the historical control arm as appropriate. Additionally, the Student t-test was used for comparing continuous variables, such as age, tumor size, and radiation dose. According to previous studies, a minimum sample size (in the study arm) of 30 patients is required to ensure $80 \%$ power with a $5 \%$ confidence interval. All statistical tests were two sided and P-values less than 0.05 were considered as statistically significant.

\section{RESULTS}

This phase-II clinical trial included 34 patients (study arm) with rectal cancer. All the patients were treated with a curative intent. The study participants included 27 males and 7 females, with the median age of 55 years (range, 26-82 years). Twenty-three patients (68\%) had clinical stage-II disease, and 11 (32\%) had clinical stage-III disease. The median clinical tumor size was $5 \mathrm{~cm}$ (range, 1.5-12 cm). Additionally, the median distance from the anal verge was $4 \mathrm{~cm}$ (range, $0-7 \mathrm{~cm}$ ). Table 1 presents the distributions of 34 patients in the study arm and the 102 patients in the historical control arm, as well as comparisons of their tumor clinical characteristics. Accordingly, no statistically significant differences were noted between the two arms in terms of the basic clinical characteristics.

All the patients in the study arm received 15-Gy LDRBT as a boost to neoadjuvant chemoradiation. LDRBT effectively and rapidly alleviated rectal pain and bleeding in all the symptomatic cases. On rectal examination, some tumor regression was observed in all the patients a week after the last session of brachytherapy; however, no clinical complete response was achieved. The patients subsequently received concurrent chemoradiation, and a median dose of 45 Gy (range, 45-50.5 Gy) was delivered within 5-6 weeks. After a median of 14 weeks following the start of intervention, all patients underwent a LAR $(n=18)$ or an APRs $(n=$ 16). Pathologic examinations showed significant tumor regression (5.2 cm vs. $2.1 \mathrm{~cm}, \mathrm{P}<0.001)$ with 10 PCRs (29.4\%) compared to

Table 1. Distribution of the patients' and the tumor clinical characteristics in 136 rectal cancer patients treated with neoadjuvant chemoradiation

\begin{tabular}{|c|c|c|c|c|}
\hline \multirow[b]{2}{*}{ Variable } & \multirow[b]{2}{*}{ Total } & \multicolumn{2}{|c|}{ Neoadjuvant chemoradiation } & \multirow[b]{2}{*}{ P-value } \\
\hline & & $\begin{array}{c}\text { With } \\
\text { brachytherapy }\end{array}$ & $\begin{array}{c}\text { Without } \\
\text { brachytherapy }\end{array}$ & \\
\hline No. of patients & 136 & 34 & 102 & \\
\hline Age (yr) & 136 & $54.9 \pm 14.6$ & $57.0 \pm 14.1$ & 0.457 \\
\hline Sex & & & & 0.501 \\
\hline Male & 100 & 27 & 73 & \\
\hline Female & 36 & 7 & 29 & \\
\hline Tumor stage & & & & 0.980 \\
\hline $\mathrm{T} 2$ & 9 & 2 & 7 & \\
\hline T3 & 115 & 29 & 86 & \\
\hline $\mathrm{T} 4$ & 12 & 3 & 9 & \\
\hline Node stage & & & & 0.662 \\
\hline Negative node & 97 & 23 & 74 & \\
\hline Positive node & 39 & 11 & 28 & \\
\hline Clinical tumor size (cm) & & $5.2 \pm 1.5$ & $5.5 \pm 2.0$ & 0.440 \\
\hline Distance from anal verge $(\mathrm{cm})$ & & $3.6 \pm 2.1$ & $4.4 \pm 2.9$ & 0.158 \\
\hline
\end{tabular}

Values are presented as number or mean \pm standard deviation. 
Table 2. The grade of tumor regression based on the Mandard's tumor regression system in the study and historical control arms

\begin{tabular}{lllr}
\hline Treatment arms & $\begin{array}{c}\text { Major response } \\
\text { (TRG 1-2) }\end{array}$ & $\begin{array}{c}\text { Minor response } \\
\text { (TRG 3-4) }\end{array}$ & $\begin{array}{r}\text { No response } \\
\text { (TRG 5) }\end{array}$ \\
\hline Study arm & $14(41 \%)$ & $19(56 \%)$ & $1(3 \%)$ \\
Control arm & $20(19.5 \%)$ & $73(71.5 \%)$ & $9(9 \%)$ \\
Total & $34(25 \%)$ & $92(68 \%)$ & $10(7 \%)$ \\
\hline
\end{tabular}

TRG, tumor regression grade.

Table 4. Rate of treatment-related toxicity in the study and control arms

\begin{tabular}{lccc}
\hline \multirow{2}{*}{ Variable } & \multicolumn{2}{c}{ Neoadjuvant chemoradiation } & \\
\cline { 2 - 3 } & $\begin{array}{c}\text { With } \\
\text { brachytherapy }\end{array}$ & $\begin{array}{c}\text { Without } \\
\text { brachytherapy }\end{array}$ & P-value \\
\hline Radiation dermatitis & 15 & 36 & 0.415 \\
Grade 1 & 19 & 66 & \\
Grade 2 & & & 0.004 \\
Proctitis & 0 & 11 & \\
Grade 0 & 26 & 86 & \\
Grade 1 & 5 & 5 & \\
Grade 2 & 2 & 0 & \\
Grade 3 & & & 0.029 \\
Noninfective cystitis & 26 & 90 & \\
Grade 0 & 6 & 12 & \\
Grade 1 & 2 & 0 & \\
Grade 2 & & & 0.015 \\
Anemia & 11 & 64 & \\
Grade 0 & 19 & 29 & \\
Grade 1 & 4 & 8 & \\
Grade 2 & 0 & 1 & \\
Grade 3 & & & \\
\hline
\end{tabular}

before the intervention. However, no significant node response was observed $(\mathrm{P}=0.232)$ compared to the initial clinical stage. Table 2 presents the grade of tumor regression based on Mandard's tumor regression system as major response (TRG 1-2), minor response (TRG 3-4), or no response (TRG 5). Accordingly, the surgical margin was free of cancer in 30 patients $(88 \%)$ and microscopically involved in four patients (12\%), 2 involving the distal margins in LAR and 2 involving the circumferential resected margins in APR. In the control arm also, seven microscopic involvements included five involving the distal margins in LAR and two involving the circumferential resected margins in APR.

Comparisons of the treatment and the pathologic tumor characteristics between the study arm and the historical control arm are been presented in Table 3. As the table depicts, a significant difference was found in terms of pathologic tumor size $(2.1 \mathrm{~cm}$ vs. 3.6 $\mathrm{cm}, \mathrm{P}=0.001)$, pathologic tumor stage (35\% T3-4 vs. $65 \% \mathrm{~T} 3-4$,
Table 3. Treatment and pathologic tumor characteristics in 136 rectal cancer patients treated with neoadjuvant chemoradiation

\begin{tabular}{|c|c|c|c|}
\hline \multirow[b]{2}{*}{ Variable } & \multicolumn{2}{|c|}{ Neoadjuvant chemoradiation } & \multirow[b]{2}{*}{ P-value } \\
\hline & $\begin{array}{c}\text { With } \\
\text { brachytherapy }\end{array}$ & $\begin{array}{c}\text { Without } \\
\text { brachytherapy }\end{array}$ & \\
\hline Dose of external RT (Gy) & $46.0 \pm 2.1$ & $47.1 \pm 2.7$ & 0.041 \\
\hline Time to surgery (wk) & $14.2 \pm 0.8$ & $11.3 \pm 0.0$ & $<0.001$ \\
\hline Type of rectal surgery & & & 1.000 \\
\hline LAR & 18 & 53 & \\
\hline APR & 16 & 49 & \\
\hline Surgical margin status & & & 0.467 \\
\hline Free & 30 & 95 & \\
\hline Involved & 4 & 7 & \\
\hline Pathologic tumor size (cm) & $2.1 \pm 2.0$ & $3.6 \pm 2.4$ & 0.001 \\
\hline Pathologic tumor stage & & & 0.003 \\
\hline T0-2 & 22 & 35 & \\
\hline T3-4 & 12 & 67 & \\
\hline Pathologic node stage & & & 0.824 \\
\hline Negative node & 24 & 75 & \\
\hline Positive node & 10 & 27 & \\
\hline Tumor grade & & & 0.392 \\
\hline I & 26 & 68 & \\
\hline$\|-\| \|$ & 8 & 34 & \\
\hline Lymphatic-vascular invasion & & & 0.837 \\
\hline No & 23 & 66 & \\
\hline Yes & 11 & 36 & \\
\hline Perineural invasion & & & 0.823 \\
\hline No & 26 & 74 & \\
\hline Yes & 8 & 28 & \\
\hline Obstruction or perforation & & & 0.464 \\
\hline No & 29 & 80 & \\
\hline Yes & 5 & 22 & \\
\hline Pathologic complete response & & & 0.028 \\
\hline No & 24 & 90 & \\
\hline Yes & 10 & 12 & \\
\hline
\end{tabular}

Values are presented as mean \pm standard deviation or number.

LAR, low anterior resection; APR, abdominoperineal resection.

$\mathrm{P}=0.003)$, and PCR $(29.4 \%$ vs. $11.7 \%, \mathrm{P}<0.028)$ in favor of the study arm. In noncomplete responder cases, we checked the tumor grade regression or progression and found tumor grade regression in three cases (one in the study arm and two in the control arm) and tumor grade progression in nine cases (three in the study arm and six in the control arm). Additionally, the dose of EBRT $(P=0.041)$ was significantly higher in the control arm, but the time to surgery was longer in the study arm $(\mathrm{P}<0.001)$. 
Regarding treatment-related toxicity, most patients developed grade- 2 dermatitis, grade- 1 proctitis, and grade- 1 anemia. However, noninfective cystitis was infrequent. Other hematologic complications, such as neutropenia and thrombocytopenia, were also rare. Treatment-related toxicities were easily tolerated, and all the patients completed their planned treatment without interruption. No significant difference was observed between the two arms regarding perioperative and early postoperative surgical complications. Table 4 presents and compares the rate of treatment-related toxicities in the study and the control arms. Accordingly, proctitis, anemia, and noninfective cystitis were significantly more frequent in the study arm compared to the control arm. In addition, perioperative and early postoperative surgical complications, such as anastomotic leakage, delayed wound healing, increase in infections, fistula formation, and neuropathy, were infrequent, and the two treatment groups did not differ significantly in this respect.

\section{DISCUSSION}

Neoadjuvant chemoradiation with a conventional fractionation of EBRT has become a standard approach for the treatment of locally advanced rectal cancer. Despite this treatment strategy, local control, sphincter preservation, and late side effects of radiation on normal tissues have remained as therapeutic challenges in patients with rectal cancer $[6,14,17]$. In resected rectal cancer, the mesorectum and the posterior and the inferior pelvis are at the highest risk for local recurrence. The risk of inferior pelvic region involvement is greatest among patients with distal rectal cancer and those who have undergone an APR [7, 18]. Furthermore, a correlation exists between local recurrence and poor overall survival. A radiation dose escalation may potentially enhance the local control rate; however, it may increase unacceptable toxicities. Therefore, endocavitary brachytherapy has good potential for use in treating patients with distal rectal cancer, particularly those who are candidates for an APR which is accompanied by a higher risk of locoregional recurrence. Endocavitary brachytherapy can be used alone or, more commonly, combined with EBRT to treat patients with rectal cancer $[8,14,19]$.

Low-voltage $(50 \mathrm{kV})$ contact $\mathrm{x}$-ray therapy is a type of endocavitary radiation therapy. The percentage depth doses for this machine are $100 \%$ at $0-\mathrm{mm}$ depth, $44 \%$ at $5 \mathrm{~mm}, 23 \%$ at $10 \mathrm{~mm}$, and $9 \%$ at $20 \mathrm{~mm}$. Therefore, this photon energy is not suitable for thick or large tumors and must be used only for early ( $\mathrm{T} 1$ and favorable 2$)$, superficial $(<5$-mm thickness), small $(<3 \mathrm{~cm})$ rectal lesions. Definitive contact $\mathrm{x}$-ray therapy can provide good local control; however, it is associated with unacceptably high rates of severe toxicity [11, 20-22]. In the Lyon 092 trial, contact radiotherapy was used as a boost following EBRT and showed an improvement in local control and sphincter preservation [14]. Currently, most radiotherapy centers use HDR brachytherapy units with iridium-192 source; these units provide better dose penetra- tion and better dose distribution in these situations. Some authors have investigated definitive endocavitary irradiation in combination with either interstitial brachytherapy or EBRT to overcome the acute and late radiation side effects on normal tissue $[12,13]$. Other investigators used neoadjuvant endorectal brachytherapy alone or in combination with EBRT in treating patients with rectal cancer.

Vuong et al. [23] evaluated the efficacy and the safety of neoadjuvant HDR brachytherapy alone in 100 patients with T2-4 (93 $\mathrm{T} 3,4 \mathrm{~T} 4$, and $3 \mathrm{~T} 2$ ) rectal cancer. The patients received 26-Gy HDR endorectal brachytherapy in four fractions. All the patients developed grade- 2 proctitis. The 5-year local control, disease-free survival, and overall survival rates were $95 \%, 65 \%$, and $70 \%$, respectively. Thus, they concluded that neoadjuvant HDR endorectal brachytherapy provided comparable oncologic outcomes and better toxicity profiles than conventional EBRT did. In another similar study, Smith et al. [24] compared the efficacy and the safety of HDR endorectal brachytherapy versus conventional chemoradiation in the neoadjuvant setting for resectable stages-II and -III low rectal cancer. In that study, 7 patients received 26-GY (in four consecutive daily fractions) neoadjuvant endorectal brachytherapy without chemotherapy while 25 historical controls undergoing 3DRT or IMRT received 28 daily 1.8-Gy fractions in concomitant 5-fluorouracil. They found similar results in terms of complete response rates, treatment-related toxicity profiles, and short-term outcomes.

Conventional neoadjuvant chemoradiation provides a $10 \%-15 \%$ PCR rate and a substantial rate of tumor down-staging $[25,26]$. In the present study, the PCR rate and the significant rate of tumor regression in the historical control arm (12\%) were comparable to the values reported in the literature. Many reports have suggested that incorporation of brachytherapy as a boost could enhance the PCR rates and improve the R0 resection rates without increasing severe toxicity [27-30]. This treatment approach may be particularly preferred for elderly patients who are not good candidates for intensive chemoradiation [31].

In a study, Sun Myint et al. [8] investigated the impact of HDR endorectal brachytherapy boost (10 Gy in 2 fractions) following preoperative chemoradiation (45 Gy/25 fractions/5 weeks) on PCR in patients with locally advanced rectal cancer. They found a $31 \%$ PCR rate in the patients receiving brachytherapy boost compared to $12 \%$ in those having conventional chemoradiation. Additionally, the rate of R0 resections was $83 \%$ in the patients who received brachytherapy boost. They found no increase in grade-3 or -4 toxicity related to radiation, no delay in wound healing, and no increase in anastomotic leakage. These results were very similar to our findings, which indicated a $29.5 \%$ PCR and an $88 \%$ R0 resection. In another study, El-Sayed and El-Taher used HDR brachytherapy boost (8 Gy in 2 fractions) following EBRT (45 Gy/25 fractions/5 weeks) concomitant with chemotherapy in 17 patients with locally-advanced rectal cancer. They found PCR in 8 patients (47\%) with an acceptable toxicity profile. Furthermore, 
grade-3 radiation proctitis (6\%) and grade- 3 dermatitis (12\%) were the most significant treatment-related toxicities [27].

In a Danish randomized phase-III trial, Jakobsen et al. [32] investigated the effectiveness of neoadjuvant chemoradiation alone ( $\operatorname{arm} \mathrm{A})$ or combined with endorectal brachytherapy ( $\operatorname{arm} B$ ) in 284 patients with resectable T3 and T4 rectal tumors. EBRT (50.4 Gy in 28 fractions) in conjunction with chemotherapy was followed by endorectal brachytherapy consisting of $10 \mathrm{~Gy}$ (in 2 fractions) prescribed at $10 \mathrm{~mm}$ from the applicator surface ( $\mathrm{arm} \mathrm{B}$ ). They found no significant difference between the two arms concerning the PCR rate ( $18 \%$ vs. $18 \%)$. However, the rate of major tumor regression ( $29 \%$ vs. $44 \%, \mathrm{P}=0.04)$ and the rate of $\mathrm{R} 0$ resection of T3 lesions ( $90 \%$ vs. $99 \%, P=0.03$ ) were significantly higher in the brachytherapy boost arm. Long-term follow-up of this clinical trial failed to show any benefit in the 5 -year progression-free and the overall survival rates [19]. However, larger studies showed that the PCR following neoadjuvant chemoradiation was associated with a lower local recurrence rate and higher fiveyear disease-free and overall survival rates. In a large European multicenter study, in the patients who had PCR following standard-course neoadjuvant chemoradiation, the local recurrence rate was only $1.6 \%$, and the five-year disease-free and overall survival rates were $85 \%$ and $90 \%$, respectively [33]. In another study of a pooled analysis including 14 different datasets, 484 out of 3,105 patients $(16 \%)$ achieved PCR. In patients with PCR, the five-year disease-free survival rate was $83 \%$ compared to $66 \%$ in those without PCR [34]. Overall, although a PCR does not necessarily translate into a better oncologic outcome, it is a potential indicator of improved locoregional control and improved diseasefree, or even overall, survival rate.

To the best of our knowledge, this is the first study to investigate the efficacy and the safety of LDRBT as a boost to neoadjuvant chemoradiation for the treatment of patients with distal rectal cancer. Additionally, this study is the first to use concurrent chemotherapy with endorectal brachytherapy in these patients. In the present study, only two patients (6\%) developed grade- 3 toxicity such as proctitis, and no severe toxicities were observed. This may be due to better biological profile of LDRBT used in our study compared to that of HDR brachytherapy used in other studies. Therefore, our study suggests that fractionated LDRBT provides a better toxicity profile than fractionated HDR endorectal brachytherapy does.

In a previous study, Lee et al. [35] showed that 4 additional weeks of neoadjuvant chemotherapy with capecitabine was safe during the resting periods before the surgery, but it did not improve the tumor response rate compared to conventional chemoradiation. A standard period of conventional neoadjuvant therapy consists of 5-6 weeks of concurrent chemoradiation, followed by a 6- to 8-week recovery window and then by a surgical resection. Therefore, the timeframe of conventional neoadjuvant therapy for patients with rectal cancer is 9-12 weeks [36]. In the current study, addition of LDRBT to EBRT prolonged the preoperative period from 9-12 weeks to 11-14 weeks. Theoretically, during the period of neoadjuvant chemoradiation, there is a potential increase in the risk of micrometastasis. Oxaliplatin is among the most effective chemotherapeutic agents for treating patients with metastatic colorectal cancer. Therefore, we added oxaliplatin to concurrent chemotherapy (capecitabine) on the first day of LDRBT (in the study arm) and EBRT (in both arms) to reduce the chance of micrometastasis.

A goal of neoadjuvant chemoradiation in patients with rectal cancer is the increasing number of sphincter-preserving operations [25]. In the current research, no statistically significant differences were found in the rates of sphincter-sparing surgery. This may be somewhat due to the locations of the rectal tumors in the patients in the study arm being lower than those in the patients in the historical control arm. Furthermore, no statistically significant differences were observed between the treatment arms regarding the distance from the anal verge.

In conclusion, a boost of LDRBT can optimize the PCR in patients with locally advanced distal rectal cancer with acceptable toxicities. In this study, beside radiation dose escalation, longer time to surgery and more effective neoadjuvant chemotherapy might have also contributed to the improvement in the PCR rate. Nevertheless, long-term follow-up is needed to translate these results to improved local control and improved overall survival rates.

\section{CONFLICT OF INTEREST}

No potential conflict of interest relevant to this article was reported.

\section{ACKNOWLEDGMENTS}

This clinical trial was approved and supported by Shiraz University of Medical Sciences (research project number 92-P-5686). This manuscript is part of a thesis by Shadi Zohourinia. Shiraz University of Medical Sciences supported this study. In addition, the study was registered on the clinical trials registration website of Iranian Registration of Clinical Trial (IRCT) on 6 December 2014 (IRCT registration number: IRCT201501059979N2). The authors would like to thank Ms. A. Keivanshekouh at the Research Improvement Center of Shiraz University of Medical Sciences for improving the use of English in the manuscript.

\section{REFERENCES}

1. Jemal A, Bray F, Center MM, Ferlay J, Ward E, Forman D. Global cancer statistics. CA Cancer J Clin 2011;61:69-90.

2. Park HC, Shin A, Kim BW, Jung KW, Won YJ, Oh JH, et al. Data on the characteristics and the survival of Korean patients with colorectal cancer from the Korea central cancer registry. Ann Coloproctol 2013;29:144-9. 
3. Hoseini S, Moaddabshoar L, Hemati S, Mohammadianpanah M. An overview of clinical and pathological characteristics and survival rate of colorectal cancer in Iran [abstract]. Ann Colorectal Res 2014;2:e17264.

4. Omidvari S, Hamedi SH, Mohammadianpanah M, Razzaghi S, Mosalaei A, Ahmadloo N, et al. Comparison of abdominoperineal resection and low anterior resection in lower and middle rectal cancer. J Egypt Natl Canc Inst 2013;25:151-60.

5. Kye BH, Cho HM. Overview of radiation therapy for treating rectal cancer. Ann Coloproctol 2014;30:165-74.

6. Kapiteijn E, Marijnen CA, Nagtegaal ID, Putter H, Steup WH, Wiggers T, et al. Preoperative radiotherapy combined with total mesorectal excision for resectable rectal cancer. N Engl J Med 2001;345:638-46.

7. van Gijn W, Marijnen CA, Nagtegaal ID, Kranenbarg EM, Putter $\mathrm{H}$, Wiggers T, et al. Preoperative radiotherapy combined with total mesorectal excision for resectable rectal cancer: 12-year follow-up of the multicentre, randomised controlled TME trial. Lancet Oncol 2011;12:575-82.

8. Sun Myint A, Mukhopadhyay T, Ramani VS, Perkins K, Snee AJ, Jelley $\mathrm{F}$, et al. Can increasing the dose of radiation by HDR brachytherapy boost following preoperative chemoradiotherapy for advanced rectal cancer improve surgical outcomes? Colorectal Dis 2010;12 Suppl 2:30-6.

9. Webster MJ, Devic S, Vuong T, Yup Han D, Park JC, Scanderbeg D, et al. Dynamic modulated brachytherapy (DMBT) for rectal cancer. Med Phys 2013;40:011718.

10. Webster MJ, Devic S, Vuong T, Han DY, Scanderbeg D, Choi D, et al. HDR brachytherapy of rectal cancer using a novel groovedshielding applicator design. Med Phys 2013;40:091704.

11. Lavertu S, Schild SE, Gunderson LL, Haddock MG, Martenson JA. Endocavitary radiation therapy for rectal adenocarcinoma: 10-year results. Am J Clin Oncol 2003;26:508-12.

12. Aumock A, Birnbaum EH, Fleshman JW, Fry RD, Gambacorta MA, Kodner IJ, et al. Treatment of rectal adenocarcinoma with endocavitary and external beam radiotherapy: results for $199 \mathrm{pa}-$ tients with localized tumors. Int J Radiat Oncol Biol Phys 2001;51: 363-70.

13. Coatmeur O, Truc G, Barillot I, Horiot JC, Maingon P. Treatment of T1-T2 rectal tumors by contact therapy and interstitial brachytherapy. Radiother Oncol 2004;70:177-82.

14. Gerard JP, Frin AC, Doyen J, Zhou FX, Gal J, Romestaing P, et al. Organ preservation in rectal adenocarcinoma (T1) T2-T3 Nx M0. Historical overview of the Lyon Sud - nice experience using contact $\mathrm{x}$-ray brachytherapy and external beam radiotherapy for 120 patients. Acta Oncol 2015;54:545-51.

15. Birnbaum EH, Ogunbiyi OA, Gagliardi G, Fry RD, Myerson RJ, Kodner IJ, et al. Selection criteria for treatment of rectal cancer with combined external and endocavitary radiation. Dis Colon Rectum 1999;42:727-33.

16. American Joint Committee on Cancer Colon and rectum. In: Edge SB, Byrd DR, Compton CC, Fritz AG, Greene FL, Trotti A, editors.
AJCC cancer staging manual. 7th ed. New York, NY: SpringerVerlag; 2010. p. 143-64.

17. Han JW, Lee MJ, Park HK, Shin JH, An MS, Ha TK, et al. Association between a close distal resection margin and recurrence after a sphincter-saving resection for $\mathrm{t} 3 \mathrm{mid}$ - or low-rectal cancer without radiotherapy. Ann Coloproctol 2013;29:231-7.

18. Roels S, Duthoy W, Haustermans K, Penninckx F, Vandecaveye V, Boterberg T, et al. Definition and delineation of the clinical target volume for rectal cancer. Int J Radiat Oncol Biol Phys 2006;65: 1129-42.

19. Appelt AL, Vogelius IR, Ploen J, Rafaelsen SR, Lindebjerg J, Havelund $\mathrm{BM}$, et al. Long-term results of a randomized trial in locally advanced rectal cancer: no benefit from adding a brachytherapy boost. Int J Radiat Oncol Biol Phys 2014;90:110-8.

20. Christoforidis D, McNally MP, Jarosek SL, Madoff RD, Finne CO. Endocavitary contact radiation therapy for ultrasonographically staged T1 N0 and T2 N0 rectal cancer. Br J Surg 2009;96:430-6.

21. Gerard JP, Chapet O, Ortholan C, Benezery K, Barbet N, Romestaing $\mathrm{P}$. French experience with contact $\mathrm{X}$-ray endocavitary radiation for early rectal cancer. Clin Oncol (R Coll Radiol) 2007;19: 661-73.

22. Gerard JP, Ayzac L, Coquard R, Romestaing P, Ardiet JM, Rocher FP, et al. Endocavitary irradiation for early rectal carcinomas T1 (T2). A series of 101 patients treated with the Papillon's technique. Int J Radiat Oncol Biol Phys 1996;34:775-83.

23. Vuong T, Devic S, Podgorsak E. High dose rate endorectal brachytherapy as a neoadjuvant treatment for patients with resectable rectal cancer. Clin Oncol (R Coll Radiol) 2007;19:701-5.

24. Smith JA, Wild AT, Singhi A, Raman SP, Qiu H, Kumar R, et al. Clinicopathologic comparison of high-dose-rate endorectal brachytherapy versus conventional chemoradiotherapy in the neoadjuvant setting for resectable stages II and III low rectal cancer. Int J Surg Oncol 2012;2012:406568.

25. Kuvshinoff B, Maghfoor I, Miedema B, Bryer M, Westgate S, Wilkes J, et al. Distal margin requirements after preoperative chemoradiotherapy for distal rectal carcinomas: are $<$ or $=1 \mathrm{~cm}$ distal margins sufficient? Ann Surg Oncol 2001;8:163-9.

26. Roh MS, Colangelo LH, O’Connell MJ, Yothers G, Deutsch M, Allegra CJ, et al. Preoperative multimodality therapy improves disease-free survival in patients with carcinoma of the rectum: NSABP R-03. J Clin Oncol 2009;27:5124-30.

27. El-Sayed ME, El-Taher ZH. Prospective phase II study of brachytherapy boost as a component of neo-adjuvant chemotherapy and external beam radiation therapy in locally advanced rectal cancer. J Egypt Natl Canc Inst 2008;20:10-6.

28. Falk AT, Claren A, Benezery K, François E, Gautier M, Gerard JP, et al. Interstitial high-dose rate brachytherapy as boost for anal canal cancer. Radiat Oncol 2014;9:240.

29. Stojanovic S, Radosevic-Jelic I, Dabic-Stankovic K, Popov I, Josifovski J. External beam radiotherapy plus brachytherapy boost in treatment of anal canal carcinoma. Acta Chir Iugosl 2007;54:33-8. 30. Sun Myint A, Lee CD, Snee AJ, Perkins K, Jelley FE, Wong H. 


\section{Coloproctology shapour Omidvari, et al.}

High dose rate brachytherapy as a boost after preoperative chemoradiotherapy for more advanced rectal tumours: the Clatterbridge experience. Clin Oncol (R Coll Radiol) 2007;19:711-9.

31. Lestrade L, De Bari B, Montbarbon X, Pommier P, Carrie C. Radiochemotherapy and brachytherapy could be the standard treatment for anal canal cancer in elderly patients? A retrospective single-centre analysis. Med Oncol 2013;30:402.

32. Jakobsen A, Ploen J, Vuong T, Appelt A, Lindebjerg J, Rafaelsen SR. Dose-effect relationship in chemoradiotherapy for locally advanced rectal cancer: a randomized trial comparing two radiation doses. Int J Radiat Oncol Biol Phys 2012;84:949-54.

33. Capirci C, Valentini V, Cionini L, De Paoli A, Rodel C, GlynneJones R, et al. Prognostic value of pathologic complete response after neoadjuvant therapy in locally advanced rectal cancer: long- term analysis of $566 \mathrm{ypCR}$ patients. Int J Radiat Oncol Biol Phys 2008;72:99-107.

34. Maas M, Nelemans PJ, Valentini V, Das P, Rodel C, Kuo LJ, et al. Long-term outcome in patients with a pathological complete response after chemoradiation for rectal cancer: a pooled analysis of individual patient data. Lancet Oncol 2010;11:835-44.

35. Lee KH, Song MS, Park JB, Kim JS, Kang DY, Kim JY. A phase II study of additional four-week chemotherapy with capecitabine during the resting periods after six-week neoadjuvant chemoradiotherapy in patients with locally advanced rectal cancer. Ann Coloproctol 2013;29:192-7.

36. Meyer J, Balch G, Willett C, Czito B. Update on treatment advances in combined-modality therapy for anal and rectal carcinomas. Curr Oncol Rep 2011;13:177-85. 